\title{
La dichotomie Nord/Sud : déconstruire une représentation spatiale influente dans le débat sur l'avenir du nucléaire en Suède
}

Teva Meyer

\section{OpenEdition}

Journals

Édition électronique

URL : http://journals.openedition.org/mappemonde/1164

DOI : 10.4000/mappemonde.1164

ISSN : 1769-7298

Éditeur

UMR ESPACE

Référence électronique

Teva Meyer, «La dichotomie Nord/Sud : déconstruire une représentation spatiale influente dans le débat sur l'avenir du nucléaire en Suède », Mappemonde [En ligne], 126 | 2019, mis en ligne le 01 avril 2019, consulté le 15 septembre 2020. URL : http://journals.openedition.org/mappemonde/1164

Ce document a été généré automatiquement le 15 septembre 2020

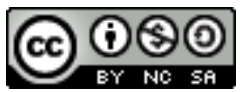

La revue Mappemonde est mise à disposition selon les termes de la Licence Creative Commons Attribution - Pas d'Utilisation Commerciale - Partage dans les Mêmes Conditions 4.0 International. 


\section{La dichotomie Nord/Sud : déconstruire une représentation spatiale influente dans le débat sur l'avenir du nucléaire en Suède}

\section{Teva Meyer}

\section{Références de la thèse}

MEYER T. (2017). Une analyse comparative des géopolitiques du nucléaire civil en Allemagne, en France et en Suède. Thèse de doctorat en Géopolitique, Université Paris 8. 


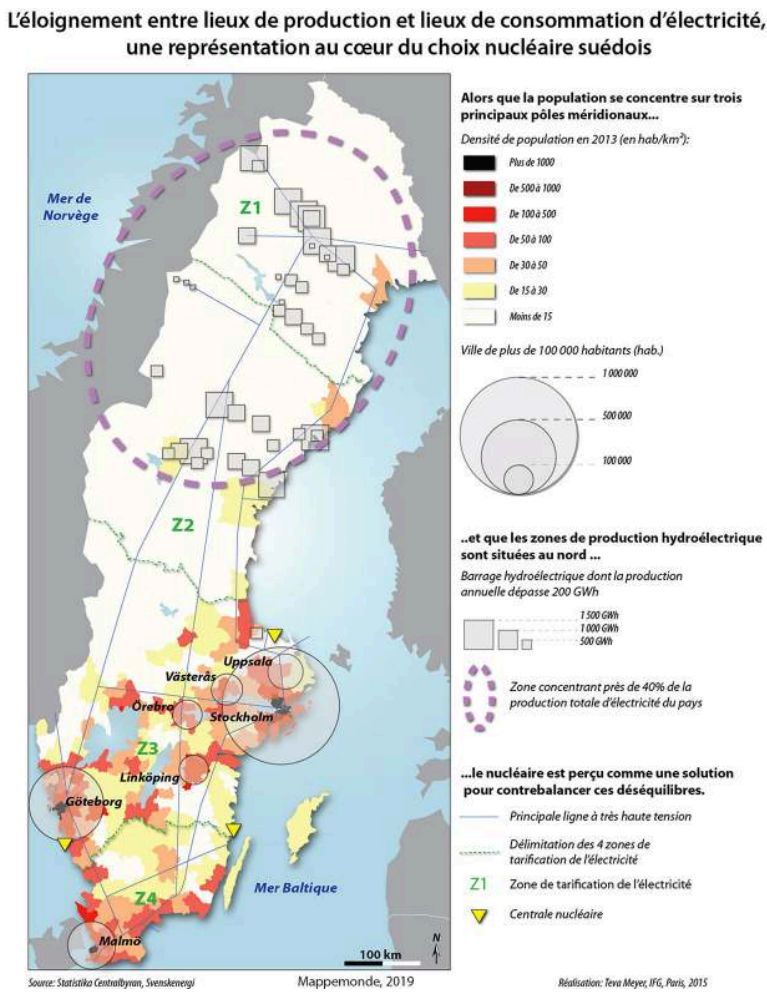

L'explosion le 11 mars 2011 des bâtiments des réacteurs 1, 2 et 3 de la centrale de Fukushima Daiichi au Japon a eu des répercussions politiques différentes dans le monde et, a fortiori, en Europe. Alors que certains pays, comme l'Allemagne, la Suisse et la Belgique, décidaient d'accélérer la fermeture de leurs centrales, d'autres continuaient la relance de leur programme atomique. C'est le cas de la Suède qui, début 2011, abrogeait le moratoire sur le nucléaire introduit en 1981, ouvrant la porte à la construction de nouveaux réacteurs. L'évolution des politiques nucléaires nationales a fait l'objet de multiples études en économie, en sociologie et en science politique. Les géographes n'ont, jusqu'à présent, pas participé à ce débat. Alors que la géographie des énergies connaît un renouveau d'intérêt (Calvert, 2015), ma thèse propose d'utiliser les outils de l'analyse spatiale pour comprendre l'évolution des politiques nucléaires.

En appliquant la méthode de la géopolitique locale (Subra, 2014), on prend le parti de considérer les politiques énergétiques comme le résultat d'un conflit entre soutiens et détracteurs des différentes sources disponibles. L'approche géopolitique considère ces conflits comme l'expression d'intérêts contradictoires qui s'accompagnent de représentations concurrentes du territoire. Afin de comprendre la relance du nucléaire en Suède, j'ai donc cherché à singulariser et à déconstruire, par des entretiens semidirectifs ainsi que par le dépouillement de la presse et de la littérature militante, les représentations spatiales qui ont structuré l'engagement des acteurs. Je propose ici de m'attarder sur la cartographie de l'une d'entre elles: l'éloignement entre les lieux de production et les lieux de consommation de l'électricité en Suède. 


\section{Les rôles de l'énergie : une analyse géopolitique des systèmes énergétiques par l'outil des représentations}

3 Les systèmes énergétiques ne servent pas qu'à fournir de l'énergie aux populations ou aux industries. L'énergie est un système technopolitique, dans le sens où des acteurs l'utilisent pour atteindre des objectifs politiques (Hecht, 1998). Les rôles donnés à l'énergie sont conditionnés par un ensemble de représentations spatiales propres à chaque pays. Les acteurs soutiennent alors la source qu'ils considèrent comme la plus apte à remplir le rôle attribué. Mes recherches ont montré que l'engagement pronucléaire en Suède était structuré par des stratégies visant à mobiliser l'énergie comme un outil d'aménagement du territoire.

De multiples travaux ont souligné l'existence d'une dichotomie ou d'un gradient Nord/ Sud en Suède dans la répartition des industries (Berger, 2012), la géographie électorale (Stegmann, 2014) ou encore la démographie (Hjort, 2006). Ces différences ont structuré les représentations que la population suédoise se fait de son propre territoire dans une opposition entre deux pôles distincts (Eriksson, 2008; Lisberg-Jansen, 2007), influençant alors les stratégies d'aménagement. La géographie de la production et de la consommation d'électricité en Suède n'y échappe pas.

\section{L'éloignement entre lieux de production et lieux de consommation d'électricité, une représentation au cœur du choix nucléaire suédois}

5 La force hydraulique constitue en moyenne $50 \%$ de l'électricité produite en Suède. Comme représenté sur la carte, près de $80 \%$ de cette production est réalisée par les grands barrages situés dans le nord. Cette concentration relève autant de facteurs naturels - les Alpes scandinaves constituent la source des principaux fleuves - que de l'histoire industrielle de la région où les barrages ont été construits pour soutenir l'industrie extractive. La géographie des populations est une image en négatif de celle de la production hydroélectrique. Comparée à d'autres États européens, la Suède est un grand pays avec une faible densité de population. Comme la carte le montre, les populations sont inégalement réparties et d'importantes disparités existent entre le nord et le sud du pays, où les aires urbaines de Stockholm, Göteborg et de Malmö concentrent $41 \%$ des Suédois.

6 L'éloignement entre ces différents pôles ne devient un argument pronucléaire déterministe qu'au travers d'une autre infrastructure - les lignes THT - sur lesquelles trois discours déterministes sont surimposés. Selon l'opérateur du réseau électrique suédois, la dichotomie Nord/Sud imposerait le nucléaire à la Suède pour des raisons techniques. Les centrales permettraient de maintenir un équilibre dans le réseau face aux barrages du nord, évitant alors des coupures dans certaines régions par période de grand froid. Si ce déséquilibre pouvait être compensé par la construction de nouvelles lignes, cette solution se heurte à une opposition environnementaliste. Alors que $68 \%$ de la Suède est couverte de forêts, le déboisement nécessaire à leur passage est considéré par les principales ONG environnementalistes comme une menace pour la biodiversité. Enfin, la dichotomie Nord/Sud contraindrait le maintien du nucléaire pour des raisons économiques. Plus un réseau est long, plus les matériaux qui le 
constituent font résistance au transfert d'électricité, entraînant des pertes sous forme de chaleur. Au total, près de 9 TWh, soit $7 \%$ de la consommation finale d'électricité du pays, sont perdus chaque année dans les réseaux, faisant de la Suède le pays européen le plus sujet à ce problème, avec la Norvège. Ces pertes sont mécaniquement répercutées sur le prix de l'électricité, amenant alors le personnel politique à soutenir le maintien du nucléaire dans le Sud où, qui plus est, le risque de coupures de courant est perçu comme un élément répulsif pour l'implantation de nouvelles industries. La division de la Suède en zones de tarification de l'électricité en 2011, suite à une plainte déposée par les producteurs danois d'électricité devant la Commission européenne, a renforcé cette représentation. Dans chacune d'entre elles, le prix de vente est déterminé en fonction du ratio entre la demande et la production d'électricité. De ce fait, les zones du sud connaissent, ponctuellement, des tarifs plus élevés que ceux des deux zones septentrionales. Cette situation a soulevé un mouvement d'opposition mené par des associations de consommateurs.

7 Dans cette configuration, l'énergie nucléaire est présentée comme un moyen de contrebalancer cette géographie perçue comme handicapante. La carte choisie ici propose une synthèse de cette stratégie. L'État suédois a milité dès le début du programme atomique pour l'implantation des centrales dans le sud, à proximité des lieux de consommation. Pour les soutiens de l'énergie atomique, fermer les centrales reviendrait à mettre en danger l'équilibre qu'elles apportent à la géographie de l'électricité. Toutefois, si elle est indéniablement présente dans les discours politiques, la représentation de l'éloignement est également forte en Suède, car elle y est sciemment mobilisée et mise en scène par des acteurs, et en premier lieu les exploitants des centrales, pour lesquels elle sert un objectif politique.

Il est de coutume de diviser l'utilisation des sources d'énergie entre un usage spécifique, c'est-à-dire l'utilisation d'une énergie dans un système qui ne pourrait fonctionner qu'avec elle, et un usage non spécifique, c'est-à-dire l'utilisation d'une énergie dans un système qui pourrait fonctionner avec différentes sources. Cet exemple permet de souligner l'existence d'usages politiques des systèmes énergétiques. Ces rôles sont conditionnés par un ensemble de représentations spatiales propres à chaque pays, inconscientes ou, au contraire, sciemment mobilisées par les acteurs dans leur discours. La compréhension de la construction des politiques énergétiques nationales passe alors irrémédiablement par l'identification et la déconstruction de ces représentations afin d'en saisir la genèse.

\section{BIBLIOGRAPHIE}

BERGER T. (2012). "Geographical location and urbanisation of the Swedish manufacturing industry, 1900-1960: evidence from a new database". Scandinavian Economic History Review, vol. 60, n 3, p. 290-308.

CALVERT K. (2015). "From energy geography to energy geographies: Perspectives on a fertile academic borderland". Progress in Human Geography, vol. 40, p. 105-125. 
ERIKSSON M. (2008). “(re)producing a « Peripheral » Region - Northern Sweden in the News". Geografiska Annaler: Series B, Human Geography, vol. 90, n 4, p. 369-388.

HECHT G. (1998). The radiance of France Nuclear Power and National Identity after World War II. Cambridge: The MIT Press, 453 p.

HJORT S. (2006). "The attraction of the rural: Characteristics of rural migrants in Sweden". Scottish Geographical Journal, vol. 122, n 1, p. 55-75.

LISBERG-JANSEN E. (2007). « La colonie interne du Norrland suédois : modèle d'une périphérie extractive ?». L'Espace politique, $\mathrm{n}^{\circ}$ 2. En ligne : https://espacepolitique.revues.org/741

STEGMANN M. (2014). “The 2014 Elections in Sweden - A New Political Landscape?”. AER News Letter, $\mathrm{n}^{\circ} 21$, p. 1-5.

SUBRA P. (2014). Géopolitique de l'aménagement du territoire, Paris : Armand Colin, 350 p. 